\title{
AVALIAÇÃO DA FORÇA MUSCULAR RESPIRATÓRIA EM INDIVÍDUOS SUBMETIDOS À HEMODIÁLISE
}

\author{
Ywia Danieli Valadares \\ Mestra em Fisioterapia. Docente do UNIFOR-MG \\ Alice Pereira Adão \\ Fisioterapeuta pelo UNIFOR-MG \\ Andreza Alves Ferreira \\ Fisioterapeuta pelo UNIFOR-MG
}

Recebido em: 04/12/2013

Aprovado em: 24/03/2014

\begin{abstract}
RESUMO
A Insuficiência Renal Crônica (IRC) é caracterizada pela perda progressiva e irreversível da função renal, na qual ocorre alteração do equilíbrio metabólico e hidroelétrico que termina em uremia, prejudicando os padrões normais de diurese com diminuição e perda da função renal. O tratamento mais utilizado para IRC atualmente é a hemodiálise. Tanto a IRC como o tratamento de hemodiálise afetam diretamente o sistema respiratório (função pulmonar), além disso, a doença cardiovascular, a uremia, a anemia, a fraqueza muscular, o sedentarismo, a desnutrição entre outros contribui para a diminuição da capacidade funcional em pacientes renais. Sendo assim, este estudo objetivou avaliar a força muscular respiratória em pacientes renais crônicos submetidos à hemodiálise, comparar os valores preditos para idade e correlacionar o tempo de tratamento por hemodiálise com a força muscular inspiratória e expiratória. Traçou-se o perfil da força muscular respiratória de 28 pacientes renais crônicos com idade entre 45 e 60 anos, submetidos à hemodiálise no Centro Nefrológico Formiguense Ltda. na cidade de Formiga-MG, utilizando a manovacuometria ( $\mathrm{Pi}$ max e $\mathrm{Pe}$ max), o questionário do nível de atividade física (IPAQ) e o questionário de identificação, sendo coletados os dados até duas horas do início da hemodiálise. Quando comparou a Pe max e a Pi max obtidas com a Pe max e a Pi max previstas obteve-se diferença estatística $(p=0,000)$, não foi observada correlação entre o tempo de tratamento por hemodiálise e a força muscular respiratória (Pi max e Pe max). De acordo com os resultados obtidos neste estudo, conclui-se que a força muscular respiratória está reduzida em pacientes submetidos à hemodiálise na amostra analisada tanto na Pi max quanto na Pe max, não apresentando correlação com o tempo de hemodiálise.
\end{abstract}

Palavras-chave: Insuficiência renal crônica. Hemodiálise. Força muscular respiratória.

\section{ASSESSMENT OF RESPIRATORY MUSCLE STRENGTH IN INDIVIDUALS UNDERGOING HEMODIALYSIS}




\begin{abstract}
Chronic Renal Failure (CRF) is a progressive and irreversible loss of kidney function, changing metabolic and hydroelectric balance causing uremia, imparing normal standards with decreased urine output and loss of renal function. Currently, the most common treatment is hemodialysis. As CRF as hemodialysis treatment affect respiratory system (lung function). In addition to, cardiovascular disease, uremia, anemia, weakness, inactivity, malnutrition and others contribute to decreased functional capacity in renal patients. So this study aimed to assess respiratory muscle strength in chronic renal failure patients undergoing hemodialysis, comparing predicted values for age and correlate hemodialysis treatment in inspiratory and expiratory muscle strength. Drew up the profile of respiratory muscle strength in 28 chronic renal patients aged 45-60 years, undergoing hemodialysis in Formiguense Neurologic Center in Formiga-MG, using a manometer ( $\mathrm{Pi}$ max and $\mathrm{Pe} \max$ ), a physical activity questionnaire (IPAQ), and identification questionnaire, which datas are up to two hours of beginning hemodialysis. When compared Pi max and Pe max obtained with PI max and PE max, it was obtained statistical difference $(\mathrm{p}=0.000)$. There was not correlation between duration of hemodialysis and respiratory muscle strength (Pi máx and Pe máx). According to the results obtained in this study, it is concluded respiratory muscle strength is reduced in patients undergoing hemodialysis as Pi máx and Pe máx, and there was not association with hemodialysis time.
\end{abstract}

Keywords: Chronic renal failure. Hemodialysis. Respiratory muscle strength.

\title{
1 INTRODUÇÃO
}

A Insuficiência Renal Crônica (IRC) é caracterizada pela perda progressiva e irreversível da função renal, na qual ocorre alteração do equilíbrio metabólico e hidroelétrico que termina em uremia, prejudicando os padrões normais de diurese com diminuição e perda da função renal (MARQUES; PEREIRA; RIBEIRO, 2005).

A hemodiálise é um processo de depuração e filtragem do sangue de substâncias do metabolismo como creatinina e a uréia, que precisam ser eliminadas do sistema circulatório devido à deficiência de filtragem dos rins, onde ocorre uma transferência de soluto entre o sangue e o líquido da diálise através do filtro de hemodiálise ou capilar, por meio de 3 mecanismos: difusão, ultrafiltração e convexão (NASCIMENTO; MARQUES, 2005).

Nas últimas décadas, tem sido amplamente estudada a força muscular respiratória que é avaliada pela pressão inspiratória máxima (Pi max) e da pressão expiratória máxima (Pe max), sendo esta técnica uma forma eficaz de avaliação de força muscular respiratória. A Pi max indica a força do músculo diafragma enquanto a Pe max é uma medida que indica a força dos músculos abdominais e intercostais. De qualquer maneira, trata-se de medidas que, quando sofrem variações, permitem-nos concluir que houve variações na força dos músculos respiratórios (COSTA et al., 2003). 
Por meio deste estudo, foi permitido traçar o perfil do sistema respiratório dos pacientes que realizam hemodiálise na cidade de Formiga -MG e auxiliar tanto na prevenção quanto na melhoria das complicações da IRC relacionada à hemodiálise.

\section{MATERIAIS E MÉTODOS}

Os indivíduos foram recrutados no Centro Nefrológico Formiguense - MG, onde são atendidos pacientes particulares conveniados e do Sistema Único de Saúde. Indivíduos de ambos os sexos com diagnóstico médico de Insuficiência Renal Crônica em tratamento por hemodiálise; na faixa etária entre 40 e 60 anos, não tabagistas, sedentários ou insuficientemente ativos segundo IPAQ.

Para a mensuração das pressões expiratórias máximas, Pi max e Pe max foi utilizado um manovacuômetro da marca Comercial Médica, devidamente calibrado. Foram realizadas 3 mensurações que não diferenciaram $20 \mathrm{~cm} \mathrm{H}_{2} \mathrm{O}$ sendo anotadas as 3 medidas e considerado o maior valor que foi analisado segundo a idade e sexo na fórmula de Neder et al. (1999).

\section{RESULTADOS E DISCUSSÃO}

Os voluntários foram recrutados no Centro Nefrológico Formiguese Ltda. na cidade de Formiga-MG. Foram avaliados 89 pacientes e, destes, foram excluídos 13 por serem tabagistas, 28 ex-tabagistas, 1 edema agudo de pulmão recente, 3 hipertensão arterial no momento da coleta, 2 por serem classificados como ativos pelo IPAC, 1 por apresentar IMC acima de $30 \mathrm{~kg} / \mathrm{m} 2,1$ cardiopata, 1 por realizar diálise peritonial, 5 por óbito e 7 por não assinarem o TCLE, totalizando uma amostra final de 27 indivíduos. Dos 27 pacientes avaliados, 15 eram do sexo feminino e 12 do sexo masculino.

Quando comparou-se a Pi max obtida com a Pi max prevista para suas idades e sexo (NEDER, et al., 1999) foi observada diferença estatística de $(\mathrm{p}=0,000)$. O mesmo foi observado para a variável Pe max $(\mathrm{p}=0,000)$.

A Pe max mostrou correlação moderada negativa com o tempo de hemodiálise $(\mathrm{R}=$ 0,353; $\mathrm{p}=0,071)$, como mostra o mesmo não ocorreu com a $\mathrm{Pi} \max$, em que não foi encontrada diferença estatística $(R=-0,229 ; p=0,251)$. 
Gráfico 1 - Pi max obtida e Pi max prevista
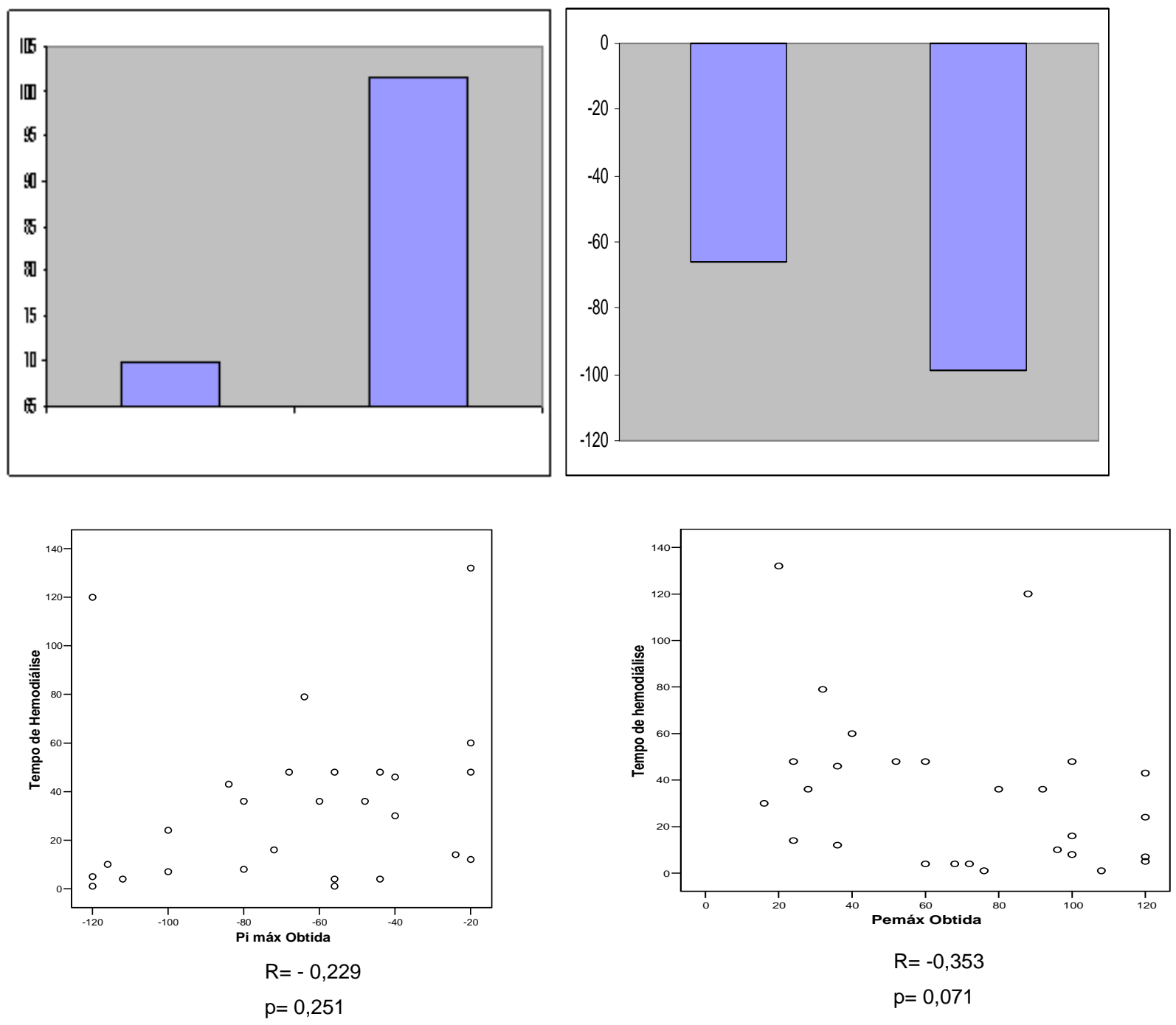

No presente estudo, pode ser observada uma predominância do sexo feminino dentre os pacientes que realizavam hemodiálise. A amostra inicial não havia diferença entre os sexos após ser aplicado o critério de exclusão de tabagistas e ex-tabagistas, o número de participantes do sexo masculino reduziu, confirmando achados que, apesar de o número de mulheres tabagistas estar aumentando, ainda há predominância em fumantes do sexo masculino. Segundo estimativas da Organização Mundial de Saúde (1997), existe, em média, 1,1 milhão de fumantes no mundo, estimando-se que $47 \%$ dos homens fumam, $12 \%$ das mulheres (INCA, 2002-2003).

Foi utilizado para medir a força muscular respiratória a manuvacuometria para obter a Pi max e Pe max devido às inúmeras vantagens. Desde a década de 60 e 70, a monuvacuometria tem sido usada para avaliar a força muscular respiratória em pacientes sadios com 
disfunção respiratória e neurológica, devido às vantagens de ser um método simples prático e preciso (COSTA, et al., 2003).

Neste estudo, foram excluídos indivíduos com IMC acima de $30 \mathrm{~kg} / \mathrm{m} 2$. Segundo Koeing (2001), a ineficácia dos músculos respiratórios, a força da musculatura respiratória e o endurance dos músculos de indivíduos obesos podem estar diminuídos quando comparado com indivíduos não obesos. O aumento do tecido adiposo pode promover uma compressão mecânica sobre os pulmões, diafragma e caixa torácica que pode gerar uma insuficiência pulmonar restritiva, o acréscimo de massa na parede torácica e na cavidade abdominal podem resultar em IMC elevado, que pode afetar a expansão do tórax gerando uma restrição e deterioração da função pulmonar (LADOSKY; BOTELHO; ALBURQUERQUE, 2001). Devido às possíveis alterações ocasionadas pela obesidade na mecânica respiratória, poderiam ser confundidas com as alterações ocasionadas pela IRC na amostra pesquisada, optou-se por excluir indivíduos obesos da amostra.

Para a coleta de dados, optou-se por restringir o tempo que o indivíduo tinha iniciado a hemodiálise naquele dia, sendo que, em todos os pacientes, a coleta foi realizada até duas horas do início da hemodiálise. Moore et al. (1998) demonstraram que, após duas horas de hemodiálise, ocorre uma descompensação cardiovascular que pode prejudicar a realização de exercícios e poderia ter influenciado na avaliação da força muscular respiratória.

Para avaliar o nível de atividade física dos indivíduos que realizavam hemodiálise, foi utilizado o IPAQ versão curta (MATSUDO, 2001). O Questionário de Atividade Física (International Physical Actinty Questionnaire - IPAQ) foi criado com a finalidade de testar e obter medidas de atividade física que fossem internacionalmente comparadas, sendo validado em 12 países e 14 centros de pesquisas. Por meio dele é possível estimar o tempo semanal gasto em atividades físicas vigorosas e moderadas, em diferentes contextos como: transporte, tarefas domésticas, trabalho e lazer e ainda o tempo gasto em atividades passivas na posição sentada.

Existe uma escassez de informações, na literatura, sobre a força muscular respiratória em indivíduos submetidos à hemodiálise. Sendo um assunto de extrema importância para detecção precoce de possíveis alterações e prevenção dessas alterações e, consequentemente, melhora da qualidade de vida desses pacientes.

\section{CONCLUSÃO}


De acordo com os resultados obtidos neste estudo, conclui-se que a força muscular respiratória está reduzida em pacientes submetidos à hemodiálise na amostra analisada tanto na Pi max quanto na Pe max, não apresentando correlação com o tempo de hemodiálise.

Neste contexto, enfatizamos a importância da inclusão do fisioterapeuta no centro de hemodiálise. Assim, a fisioterapia irá atuar na prevenção de tais complicações, por intermédio de exercícios de fortalecimento dos músculos respiratórios, técnicas manuais e instrumentais, exercícios, posicionamento, reeducação e orientações, proporcionando uma mecânica respiratória eficiente para uma melhor qualidade de vida dos indivíduos.

\section{REFERÊNCIAS}

ABENSUR, H. Uso da diálise peritoneal em pacientes com insuficiência cardíaca congestiva. Revista Brasileira de Hipertensão, São Paulo, v. 15, n. 3, p. 162-165, 2008.

ABREU, I. S. Qualidade de vida relacionada a saúde de pacientes em hemodiálise no Município de Guarapuera-PR. 2005. 87 f. Dissertação (Mestrado em Enfermagem em Saúde pública) - Universidade de São Paulo-Escola de Enfermagem de Ribeirão Preto, Ribeirão Preto, 2005.

AIRES, M. de M. Fisiologia. 2. ed. Rio de Janeiro: Guanabara Koogan, 1999.

AZEREDO, C. A. C. Fisioterapia respiratória moderna. 4. ed. São Paulo: Manole, 2002.

BARDIM, T. Musculos keletal manifestations of chronic renal failure. Current Opinion in Rheumatology, Cleveland, USA, v. 15, p. 48-54, 2003.

BARK, H. et al. Effect of chronic renal failure on respiratory muscle strength. Respiration, USA, v. 54, n. 3, p. 153-161, 1988.

BARROS E. M.; TOMÉ F. S.; GONÇALVES L. E. S. Nefrologia rotinas, diagnóstico e tratamento. Porto Alegre: Artmed, 2000.

BASTOS, M. G. Doença renal crônica: problemas e soluções. Jornal Brasileiro de Nefrologia, Juiz de Fora, v. 26, p. 202-215, dez. 2004.

BAUTISTA, J. et al. Dialysis myopathy: report of 13 cases. Acta Neuropathologica, Basel, Switzerland, v. 61, n. 1, p. 71-75, 1983.

BENEDIT, T. R. B. et al. Reprodutibilidade e validade do questionário internacional de atividade física (IPAQ) em homens idosos. Revista Brasileira de Medicina do Esporte, São Paulo, v. 13, n. 1, p. 10-16, jan./fev. 2007.

BLACK, L. F.; HYATT, R. E. Maximal respiratory pressures: normal values and relationship to age and sex. American Review of Respiratory Disease, New York, v. 99, n. 5, p. 696702, 1969. 
BROYER, M.; DELAPORTE, C.; MAZIERE, B. Water electrolytes and protein content of muscle obtained by needle biopsy in uremia children. Biomedicine, India, v. 38, p. 278-285, 1974.

BRUNETTO, A. F.; FREGONEZI, G. A.; PAULIN, E. Comparação das medidas das Pressões Respiratórias Máximas (PI Max, PE Max) aferidas através de manuovacuômetro e sistema de aquisição de dados. Revista Brasileira de Atividade Física e Saúde, Pelotas, v. 5, n. 2, p. 30-37, 2000.

BUSH, A.; GABRIEL, R. Pulmonary function in chronic renal failure: effects of dialysis an transplantation. Thorax, London, v. 46, p. 424-428, 1991.

CASTRO, M. et al. Qualidade de vida de pacientes com insuficiência renal crônica em hemodiálise avaliado através do instrumento genérico SF 36. Revista da Associação Médica Brasileira, São Paulo, v. 49, p. 245-249, 2003.

CASTRO, M. C. M. de. Atualização em diálise: complicações agudas em hemodiálise. Jornal Brasileiro de Nefrologia, São Paulo, v. 23, p. 108-113, 2001.

CENSOS. Disponível em:

<http://www.sbn.org.br/Censo/2008/SBN_Censo_Diálise_2008.doc>. Acesso em: 06 ago. 2009.

CESARINO, C. B.; CASAGRANDE, L. D. Paciente com insuficiência renal crônica em tratamento hemodialítico: atividade educativa do enfermeiro. Revista Latino-americana de Enfermagem, Ribeirão Preto, v. 6, n. 4, p. 31- 40, 1998.

CONSTANZO, L. S. Fisiologia. Rio de Janeiro: Guanabara Koogan, 1999.

COSTA, D. et al. Avaliação da força muscular respiratória em amplitudes torácicas e abdominais após RFR em indivíduos obesos. Revista Latino-americana de Enfermagem, Ribeirão Preto, v. 11, n. 2, p. 156-160, mar./abr. 2003.

CUNHA, M. S. et al. Avaliação da capacidade funcional da qualidade de vida em pacientes renais crônicos submetidos a tratamento hemodialítico. Revista de Fisioterapia e Pesquisa, São Paulo, v. 16, n. 2, p. 155-160, abr./jun. 2009.

DÂNGELO, J. G.; FATTINI, C. A. Anatomia humana básica. 6. ed. São Paulo: Atheneu, 2000.

FALCÃO, T. J. de O.; COSTA, I. do C. C. O tabagismo em um município de pequeno porte: estudo etnográfico com base para geração de um programa de saúde pública. Jornal Brasileiro de Pneumologia, Brasília, v. 34, n. 2, p. 91-97, 2008.

FIZ, J. A.; TEXIDÓ, A.; IZQUIERDO, J.; RUIZ, J.; ROIG, J.; MORERA, J. Postural variation of the maximum inspiratory and expiratory pressures in normal subjects. Chest Journal, USA, v. 97, n. 2, 1990.

GUYTON, A. C. Fisiologia humana. 6. ed. Rio de Janeiro: Guanabara Koogan, 1988. 
HAUTMANN, H. et al. Maximal inspiratory mouth pressures (PI Max) in healthy subjects what is the lower limit of normal. Respiratory Medicine, USA, v. 94, p. 689-693, 2000.

IKIZLER, T. A. et al. Hemodialysis stimulates muscle and whole body protein loss and alters substrate oxidation. The American Journal of Physiology - Endocrinology and Metabolism, v. 282, n. 1, p. 107-116, 2002.

JATOBÁ, J. P. C. et al. Avaliação da função pulmonar, força muscular respiratória e teste de caminhada de 6 min em pacientes portadores de DRC em hemodiálise. Jornal Brasileiro de Pneumologia, Brasília, v. 30, n. 4, p. 280-287, 2008.

KARACAN, O. et al. Pulmonary function in uremic patients on long-term hemodialysis. Renal Failure, London, v. 26, n. 3, p. 273-278, 2004.

KISNER, C.; COLBY, L. A. Exercícios terapêuticos: fundamentos e técnicas. 3. ed. São Paulo: Manole, 1998.

KOENIG, S. M. Pulmonary complications of obesity. The American Journal of the Medical Sciences, USA, v. 321, n. 4, p. 249-279, 2001.

KOVELIS, D. et al. Função pulmonar e força muscular respiratória em pacientes com doença renal crônica submetidos à hemodiálise. Jornal Brasileiro de Pneumologia, Brasília, v. 34, n. 11, p. 907-912, 2008.

KUSUMOTA, L. Avaliação da qualidade de vida relacionada à saúde de pacientes em hemodiálise. 2005. Tese (Doutorado em Enfermagem) - Escola de Enfermagem de Ribeirão Preto-Universidade de São Paulo, Ribeirão Preto, 2005.

LADOSKY, W.; BOTELHO, M. A. M.; ALBURQUERQUE JR, J. P. Chest mechanics in morbidly obesenan-hypoventilated patients. Respiratory Medicine, USA, v. 95, p. 281-286, 2001.

LARSON, J. L. et al. Discontinuos incremental threshold loading test measurement of respiratory muscle endurance in patients with COPD. Chest Journal, USA, v. 115, p. 60-67, 1999.

MALTA, D. C. et al. Prevalência do tabagismo em adultos residentes nas capitais dos estados e no Distrito Federal, Brasil, 2008. Jornal Brasileiro de Pneumologia, Brasília, v. 36, n. 1, p. 75-83, 2010.

MANSUR, H. N.; LIMA, J. R. P.; NOVAES, J. da S. Nível de atividade física em risco cardiovascular em pacientes com Doença Renal Crônica. Jornal Brasileiro de Pneumologia, Brasília, v. 29, n. 4, p. 209-214, 2007.

MARQUES, A. B.; PEREIRA, D. C.; RIBEIRO, R. C. H. M. Motivo e frequência de internação dos pacientes com IRC em tratamento hemodialítico. Arquivos de Ciências da Saúde, Ribeirão Preto, v. 12, p. 67-72, 2005.

MATSUDO, S. et al. Questionário internacional de atividade física (IPAQ): estudos de validade e reprodutividade no Brasil. Revista Brasileira de Atividade Física e Saúde, Pelotas, v. 6, n. 2, p. 05-18, 2001. 
MINISTÉRIO DA SAÚDE/SVS. Instituto Nacional do Câncer (INCA). 2002-2003. Disponível em: <http//www.datasus.gov.br/idbs>. Acesso em: 06 jun. 2010.

MOORE, G. E. et al. Cardiovascular response to submaximal stationary cycling during hemodialysis. American Journal of Kidney Diseases, USA, v. 31, p. 631-637, abr. 1998.

NAGHETTINI, A. et al. Soro prevalência do vírus da hepatite $C$ na população em diálise em Goiânia-GO. Revista da Sociedade Brasileira de Medicina Tropical, Uberaba, v. 30, n. 2, p. 113-117, mar./abr. 1997.

NASCIMENTO, C. D.; MARQUES, I. R. Intervenções de enfermagem nas complicações mais frequentes durante a sessão de hemodiálise: revisão de literatura. Revista Brasileira de Enfermagem, Santo Amaro, v. 6, n. 58, p. 719-722, 2005.

NEDER, J. A et al. Reference values for lung function tests. II. Maximal respiratory pressures and voluntary ventilation. The Brazilian Journal of Medical and Biological Research, Ribeirão Preto, v. 32, n. 6, p. 719-728, 1999.

OH-PARK, M. et al. Exercise for the dialyzed: aerobic and streng training during hemodialysis. American Journal of Physical Medicine and Rehabilitation, USA, v. 81, p. 814-821, 2002.

OS RINS e suas doenças. Disponível em: <http://www.sbn.org.br/Romão/2004/SBN _Romão_2004.doc>. Acesso em: 06 ago. 2009.

PEREIRA, M. G. Epidemiologia: teoria e prática. Rio de Janeiro: Guanabara Koogan,1995.

PREZANT, D. J. Effect of uremia and its treatment on pulmonary function. Lung, New York, v. 168, p. 1-14, 1990.

QUEIROZ, L. de O.; NASCIMENTO, R. G. do. Repercussões da hemodiálise na função respiratória de pacientes portadores de insuficiência renal crônica. 2006. $91 \mathrm{f}$. Trabalho de conclusão de curso (Graduação em Fisioterapia)-Universidade da Amazônia-UNAMA, Belém, 2006.

REBORETO, M. de M. et al. Exercício físico em pacientes dialisados. Revista de Medicina do Esporte, São Paulo, v. 13 n. 5, p. 427- 430, 2007.

RIBEIRO, R. de C. H. M. et al. Caracterização e etiologia da insuficiência renal crônica em unidade de nefrologia do interior do estado de São Paulo. Acta Paulista de Enfermagem, São Paulo, v. 21, n. especial, p. 207-211, 2008.

ROCHA, C. B. Avaliação das pressões respiratórias máximas de pacientes renais crônicos submetidos à hemodiálise. 2008. 83 f. Tese (Doutorado em Cirurgia)-Faculdade de Ciências Medicas da UNICAMP, Campinas, 2008.

ROCHA, C. B.; ARAUJO, S. Avaliação das pressões respiratórias máximas em pacientes renais crônicos nos momentos pré e pós hemodiálise. Jornal Brasileiro de Nefrologia, Brasília, v. 32, n. 1, p. 107-117, jan./fev./mar. 2010. 
ROMÃO, J. E. J. et al. Censo SBN 2002: informações epidemiológicas das unidades de diálise do Brasil. Jornal Brasileiro de Nefrologia, São Paulo, v. 4, n. 25, p. 188-199, 2002.

SATHVIK, B. S. et al. Medication knowledge of hemodialysis patients and influence of clinical pharmacist education provided on their knowledge. Indian Journal of Pharmaceutical Sciences, India, v. 69, n. 2, p. 232-239, 2007.

SCANLAN, C. L.; WILKINS, R.L.; STOLLER, J.K. Fundamentos da terapia respiratória de Egan. 7. ed. São Paulo: Manole, 2000.

SCHARDONG, T. J.; LUKRAFKA, J. L.; GARCIA, V. D. Avaliação da função pulmonar e da qualidade de vida em pacientes com doença renal crônica submetidos à hemodiálise.

Jornal Brasileiro de Nefrologia, Porto Alegre, v. 30, n. 1, p. 40-47, 2006.

SESSO, R. Epidemiologia da insuficiência renal crônica no Brasil. In: ALZEN, H.; SCHOR, N. Guias de medicina ambulatorial e hospitalar. Barueri: Manole, 2002. cap. 1, p. 1-7.

SOUZA, R. B. de. Pressões respiratórias estáticas máximas. Jornal Brasileiro de Pneumologia, Brasília, v. 28, n. 3, out. 2002.

STEINHORST, R. C. Influência dos procedimentos hemodialíticos na mecânica respiratória em pacientes com insuficiência renal, aguda ou crônica, sob ventilação mecânica invasiva. 2005. 67 f. Dissertação (Mestrado em Ciências)-Universidade de São Paulo, São Paulo, 2005.

TRENTINI, M. et al. Qualidade de vida de pessoas dependentes de hemodiálise considerando alguns aspectos físicos, sociais e emocionais. Revista Texto e Contexto Enfermagem, v. 13, n. 1, p. 74-82, 2004.

TORTORA, G. J. Corpo humano: fundamentos de anatomia e fisiologia. São Paulo: Artmed, 2000.

VIEIRA, W. P. et al. Manifestações músculo esqueléticas submetidas à hemodiálise. Revista Brasileira de Reumatologia, São Paulo, v. 45, n. 6, p. 357-364, nov./dez. 2005.

WEINER, P. G. R.; ZAMIR, D.; ZONDER, H. Specific inspiratory muscle training in chronic hemodialysis. Harefuah, USA, v. 15, n. 130, p. 73-76, Jan. 1996.

WELCH, J. L. et al. Pattems of interdialytic weight gain during the first year of hemodialysis. Nephrology Nursing Journal, v. 33, n. 5, p. 493-499, 2006.

WILLET, W. Nutritional Epidemiology. $2^{\text {nd }}$ ed. New York: Oxford University Press, 1998. 